\title{
Análise Algébrica de Transferências Orbitais Sujeitas a Desvios Superpostos em "Pitch" e "Yaw"
}

\author{
Algebraic Analysis of Orbital Transfers Under \\ "Pitch" and "Yaw" Superposed Deviations
}

\author{
A.D.C. de Jesus* \\ Departamento de Física - UEFS \\ Campus Universitário, Km 03 BR 116 \\ Feira de Santana - BA - 44031-460
}

\begin{abstract}
Os efeitos de desvios em direção do vetor empuxo em manobras de transferência orbital de um veículo espacial podem gerar desalinhamentos definitivos, afastando-o da sua trajetória final nominal. Manobras de correção são implementadas, mas estes desvios tendem a aumentar durante o tempo de vida do veículo pelo desgaste do sistema propulsor. As manobras de correção, neste período, não conseguem alcançar seus objetivos e o veículo é perdido, devido as forças dissipativas e os desvios do sistema propulsor. O entendimento de como estes desvios interferem na órbita final é de grande importância para o controle de uma missão sujeita a desvios em "pitch" e "yaw". Neste trabalho, mostramos a relação algébrica e existente entre os desvios nestes ângulos e seus efeitos em elementos keplerianos importantes que definem a órbita final do veículo. Esta análise permite o estabelecimento teórico e exato entre os valores médios destas grandezas numa relação de causa e efeito não linear, que prevê os casos de superposição dos efeitos na direção de queima nos propulsores. Os efeitos das forças dissipativas foram considerados desprezíveis em relação aos efeitos destes desvios durante a manobra de transferência orbital.
\end{abstract}

Palavras-chaves: "Pitch", "Yaw", Desvios de Direção, Superposição, Relação NãoLinear.

The direction deviation effects of the thrust vector in space vehicle orbital transfers cause misalignment deviations with respect to its nominal action line (nominal trajectory). The correction maneuvers are implemented, but these deviations increasing during the propulsion system operation, due their consuming. The correction maneuvers during this action are inefficient and the vehicle is lost, due the non-conservative forces and the propulsion system deviations. The knowledge of these deviations effects in the final orbit is very important to space mission control under "pitch" and "yaw" deviations. In this paper, we show the algebraic relationship between these deviations and their effects in the important keplerian elements of the final orbit. This analysis provides the theoretical and exact media values of these variables through non-linear cause/effect relationship. This relationship anticipates the superposition cases of these effects in the burn direction. We considered the non-conservative forces effects practically null with respect to the direction thrust deviations effects during the orbital transfer maneuvers.

Key-words: Pitch, Yaw, Thrust Deviations, Superposed, Nonlinear Relation.

\section{INTRODUÇÃO}

O estudo de manobras orbitais de veículos espaciais para atender a diversas necessidades científicas, civis e tecnológicas teve seus primeiros passos em Goddard (1919) [1]. O problema de Goddard consistiu em maximizar a altitude final para uma ascensão vertical de um foguete sob a influência de um campo gravitacional com lei do inverso do quadrado da distância e de um arrasto atmosférico, da forma mais econômica possível. Em seguida, Hohmann (1925) [2] obteve a solução com combustível mínimo do problema da transferência bi-impulsiva de um veículo espacial entre duas órbitas circulares coplanares. Esta solução foi considerada a solução final do problema até Hoelker e Silber (1959) [3] mostrarem que a solução de Hohmann seria de mínimo combustível, apenas quando a razão entre os raios das órbitas final e inicial fosse menor que

*Endereço Eletrônico: adj@uefs.br
11,94, para uma manobra bi-impulsiva. Numa manobra não-impulsiva é de extrema importância que se conheça o ponto de aplicação do empuxo para que se evite efeitos de "espalhamento do empuxo". Lawden (1955) [4] buscou direções ótimas para a aplicação do empuxo e mostrou que a direção do empuxo deveria ser tangencial à trajetória. Existem tipos de manobras tri e multi-impulsivas sujeitas à condição de mínimo consumo de combustível e também a outras restrições, que foram estudadas por outros autores com métodos diversos. Podemos citar alguns trabalhos recentes realizados na área de Otimização de Trajetória: Prado (1989) [5], Rodrigues (1991) [6], Santos-Paulo (1998) [7], Schultz (1997) [8].

Estudar os efeitos da superposição de desvios no vetor empuxo em veículos espaciais é uma tarefa muito significativa, devido a sua importância tecnológica e também da viabilidade das missões espaciais, promovidas por veículos movidos a propulsores. Veículos que operam com órbitas mal modeladas e não otimizadas podem desviar-se dos seus alvos, tornando seu resgate impraticável devido ao custo operacional e da disponibilidade do combustível a bordo. As órbitas de satélites sujeitos a um sistema de propulsor não ideal são afetadas pelos desvios não superpostos 
de direção. Este resultado foi verificado por Jesus (1999) [9] para manobras planares de transferência orbital. Foram realizadas análises numérica e algébrica e, para manobras de transferência orbital fora do plano, foi realizada análise numérica. Logo após, foi realizada a análise numérica para desvios superpostos, desvios correlacionados por Jesus e Santos (2004a,b) [10, 11], e análise da viabilidade de uma missão espacial sob influência destes desvios por Jesus et al. (2004c) [12]. Neste trabalho realizamos a demonstração algébrica para os desvios superpostos em "pitch" e em "yaw" para as manobras de transferência orbital, estabelecendo uma relação de causa e efeito com os desvios ocorridos no semi-eixo maior.

\section{FORMALISMO MATEMÁTICO}

O problema matemático a ser resolvido é encontrar as equações do movimento do veículo espacial e mostrar algebricamente a relação de causa e efeito entre os desvios superpostos nas direção de "pitch", $\alpha$, e "yaw", $\beta$, com o desvio no semi-eixo maior da trajetória de transferência orbital do veículo. Além disso, as manobras foram realizadas com custo mínimo de combustível. A Figura 1, abaixo mostra o sistema de referência a partir do qual foram escritas as leis de Newton.

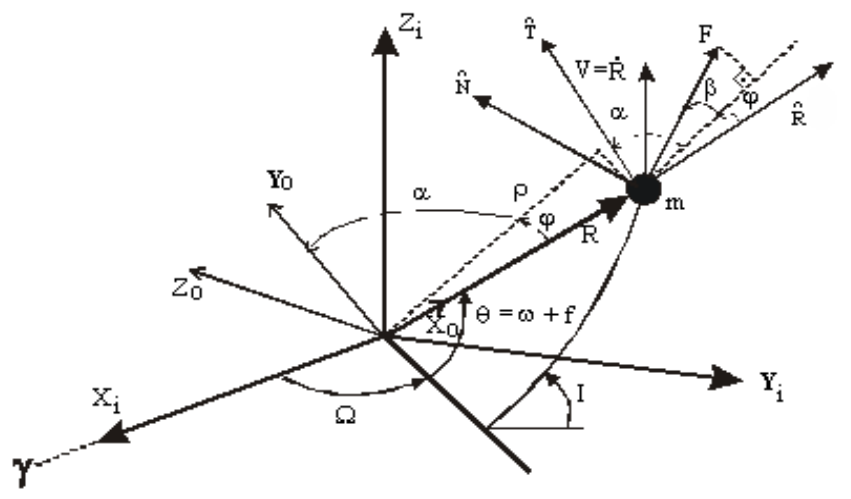

Fig. 1 - Sistemas de referência inercial e RTN

As equações do movimento do veículo neste sistema são:

$$
\begin{aligned}
& m \frac{d^{2} X}{d t^{2}}=-\frac{\mu m X}{R^{3}}+F_{X}, \\
& m \frac{d^{2} Y}{d t^{2}}=-\frac{\mu m Y}{R^{3}}+F_{Y}, \\
& m \frac{d^{2} Z}{d t^{2}}=-\frac{\mu m Z}{R^{3}}+F_{Z},
\end{aligned}
$$

$$
\begin{aligned}
F_{X}= & F[\cos \beta \operatorname{sen} \alpha(\cos \Omega \cos \theta-\cos I \operatorname{sen} \Omega \operatorname{sen} \theta) \\
& +\operatorname{sen} \beta \operatorname{sen} \Omega \operatorname{sen} I-\cos \beta \cos \alpha(\cos \Omega \operatorname{sen} \theta \\
& +\cos I \operatorname{sen} \Omega \cos \theta)], \\
F_{Y}= & F[\cos \beta \operatorname{sen} \alpha(\operatorname{sen} \Omega \cos \theta+\cos I \cos \Omega \operatorname{sen} \theta) \\
& -\operatorname{sen} \beta \cos \Omega \operatorname{sen} I-\cos \beta \cos \alpha(\operatorname{sen} \Omega \operatorname{sen} \theta \\
& -\cos I \cos \Omega \cos \theta)], \\
F_{Z}= & F[\cos \beta \operatorname{sen} \alpha \operatorname{sen} I \operatorname{sen} \theta \\
& +\cos \beta \cos \alpha \operatorname{sen} I \cos \theta+\operatorname{sen} \beta \cos I],
\end{aligned}
$$

Adotando uma dinâmica com variação linear de massa (a seguir), nas equações do movimento deverá ser incluído o termo de variação de massa.

$$
\begin{aligned}
m(t) & =m\left(t_{0}\right)+\frac{d m}{d t}\left(t-t_{0}\right), \quad \text { com } \quad \frac{d m}{d t}<0, \\
F & \approx\left|\frac{d m}{d t}\right| c .
\end{aligned}
$$

Nas coordenadas radial $R$, transversal $T$ e binormal $N$, temos

$$
\begin{aligned}
m a_{R}(t) & =F \cos \beta(t) \operatorname{sen} \alpha(t)-\frac{\mu m}{R^{2}(t)} \\
m a_{T}(t) & =F \cos \beta(t) \cos \alpha(t) \\
m a_{N}(t) & =F \operatorname{sen} \beta(t)
\end{aligned}
$$

$$
\begin{aligned}
a_{R}(t)= & \frac{d V_{R}}{d t}-\frac{V_{T}^{2}}{R}-\frac{V_{N}^{2}}{R}, \\
a_{T}(t)= & \frac{d V_{T}}{d t}+\frac{V_{R} V_{T}}{R}-V_{N} \frac{d l}{d t} \cos \theta \\
& -V_{N} \frac{d \Omega}{d t} \operatorname{sen} l \operatorname{sen} \theta, \\
a_{N}(t)= & \frac{d V_{N}}{d t}+\frac{V_{R} V_{T}}{R}+V_{T} \frac{d l}{d t} \cos \theta \\
& +V_{T} \frac{d \Omega}{d t} \operatorname{sen} l \operatorname{sen} \theta,
\end{aligned}
$$

$$
\begin{aligned}
V_{R} & =\frac{d R}{d t} \\
V_{T} & =R\left(\frac{d \Omega}{d t} \cos l+\frac{d \theta}{d t}\right) \\
V_{N} & =R\left(-\frac{d \Omega}{d t} \operatorname{sen} l \cos \theta+\frac{d l}{d t} \operatorname{sen} \theta\right), \\
\theta & =\omega+f
\end{aligned}
$$

onde $V_{N}, V_{T}$, e $V_{R}$ são as velocidades normal, transversal e radial, respectivamente. Suas derivadas são as respectivas acelerações.

A relação de causa e efeito entre os desvios no vetor empuxo e o semi-eixo maior da órbita final pode ser encontrada, a partir da consideração de que a energia mecânica do veículo varia devido o empuxo que é aplicado pelo sistema propulsor. Neste caso, o empuxo é tomado como uma força não natural em relação à força de interação gravitacional entre as massas do veículo e da terra, ambas tomadas como punctuais. Além disso, assumimos que o empuxo é não ideal em módulo e em direção, transferindo os desvios de magnitude e no apontamento (alinhamento) à dinâmica do veículo espacial. A variação da energia mecânica do veículo espacial no tempo é igual à integral das potências trocadas pelas componentes das forças resultantes nas direções $R, T$ e $N$. Assim,

$$
\begin{aligned}
\frac{d E_{m}(t)}{d t}= & F \operatorname{sen} \alpha(t) \cos \beta(t) V_{R}(t) \\
& +F \cos \alpha(t) \cos \beta(t) V_{T}(t) \\
& +F \operatorname{sen} \beta(t) V_{N}(t)
\end{aligned}
$$

Durante o intervalo de tempo $t$, calculamos a variação desta energia, 


$$
\begin{aligned}
\triangle E_{m}\left(t_{1}, t_{2}\right)=E_{m}\left(t_{2}\right)-E_{m}\left(t_{1}\right) & =\int_{t 1}^{t_{2}} F\left[\operatorname{sen} \alpha(t) \cos \beta(t) V_{R}(t)+\cos \alpha(t) \cos \beta(t) V_{T}(t)+\operatorname{sen} \beta(t) V_{N}(t)\right] d t \\
& =-\frac{\mu m}{2 a\left(t_{2}\right)}+\frac{\mu m}{2 a\left(t_{2}\right)}
\end{aligned}
$$

$a\left(t_{i}\right)$ é o semi-eixo maior da órbita do veículo no instante $i$. Esta variação da energia mecânica, dada pela equação
(20), pode ser calculada para órbitas sujeitas a desvios em "pitch", $\triangle \alpha$, e em "yaw", $\triangle \beta$ e é dada por,

$$
\begin{aligned}
\triangle E_{m}^{\prime}\left(t_{1}, t_{2}\right)=E_{m}^{\prime}\left(t_{2}\right)-E_{m}^{\prime}\left(t_{1}\right) & =\int_{t 1}^{t_{2}} F[\cos (\beta(t)+\triangle \beta(t)) \operatorname{sen}(\alpha(t)+\triangle \alpha(t))] V_{R} d t \\
& +\int_{t 1}^{t_{2}} F[\operatorname{sen}(\beta(t)+\triangle \beta(t))] V_{N}^{\prime} d t \\
& +\int_{t 1}^{t_{2}} F[\cos (\beta(t)+\triangle \beta(t)) \cos (\alpha(t)+\triangle \alpha(t))] V_{T}^{\prime} d t
\end{aligned}
$$

Ao termos em (') indicam que os desvios no vetor empuxo afetaram a referida grandeza. Definindo uma variação da variação da energia mecânica, $\triangle_{2} E_{m}\left(t_{1}, t_{2}\right)$, como sendo entre os seus valores com e sem os desvios nos ângulos "pitch" e "yaw", utilizando um pequeno algebrismo, temos,

$$
\begin{aligned}
\triangle_{2} E_{m}^{\prime}\left(t_{1}, t_{2}\right)= & \triangle E_{m}^{\prime}\left(t_{1}, t_{2}\right)-E_{m}^{\prime}\left(t_{1}, t_{2}\right)=-\frac{\mu m}{2 a^{\prime}\left(t_{2}\right)}+\frac{\mu m}{2 a^{\prime}\left(t_{1}\right)}+\frac{\mu m}{2 a^{2}\left(t_{2}\right)}-\frac{\mu m}{2 a^{2}\left(t_{1}\right)} \\
& =\int_{t 1}^{t_{2}} F\left[\operatorname{sen}(\alpha(t)+\triangle \alpha(t)) \cos (\beta(t)+\triangle \beta(t)) V_{R}^{\prime}(t)-\operatorname{sen} \alpha(t) \cos \beta(t) V_{R}(t)\right] d t \\
& +\int_{t 1}^{t_{2}} F\left[\operatorname{sen}(\beta(t)+\triangle \beta(t)) V_{N}^{\prime}(t)-\operatorname{sen} \beta(t) V_{N}(t)\right] d t \\
& +\int_{t 1}^{t_{2}} F\left[\cos (\alpha(t)+\triangle \alpha(t)) \cos (\beta(t)+\triangle \beta(t)) V_{T}^{\prime}(t)-\cos \alpha(t) \cos \beta(t) V_{T}(t)\right] d t
\end{aligned}
$$

\section{CASO NÃO CORRELACIONADO (WHITE-NOISE) ENTRE VELOCIDADE E DESVIOS}

As equações obtidas até agora são gerais, contudo, para realizarmos as integrações necessitamos admitir algumas condições que as grandezas envolvidas podem satisfazer numa missão espacial real. Neste sentido, vamos admitir que os desvios em direção não possuem correlação no tempo com as velocidades radial, transversal e normal. Isto significa que enquanto o tempo passa, os desvios no vetor empuxo não possuem uma dependência funcional com a velocidade do veículo. Além disso, admitiremos também que o semi-eixo maior no instante inicial das órbitas de partida e de chegada são iguais. Esta condição é fisicamente razoável, visto que no instante inicial não houve "tempo" para os desvios afetarem o semi-eixo maior.

Para encontrarmos esta relação de causa e efeito, aplicaremos o operador esperança $E\{x\}$, ou primeiro momento, na expressão em (20), ou média. Assim, estaremos selecio- nando os valores médios das variações das grandezas envolvidas. Na realidade, estamos admitindo que a natureza não se comporte para este fenômeno de maneira determinística, mas probabilística, na qual as médias serão boas representantes do fenômeno dinâmico, sujeito a desvios definidos a partir de uma função de probabilidade. Tecnologicamente falando, esta suposição é plausível, porque os desvios no apontamento (ou direção) de um vetor empuxo são devidos a diversas fontes, desde o deslocamento do centro de massa do veículo, devido ao gasto de combustível ou até de partes móveis (antenas, painéis solares, etc.) até desvios angulares ocasionados durante a montagem. Estes desvios e outros na magnitude do empuxo causam uma força resultante não ideal que não passa pelo centro de massa do veículo, gerando torques indesejáveis que desviam-no das suas direções nominais. As implementações tecnológicas têm mostrado que estes desvios podem ser modelados a partir de funções de probabilidade uniforme e gaussiana. A não correlação permite separar a esperança do produto de termos em um produto das esperanças destes termos. Assim, depois de algum alge- 
brismo, temos,

$$
\begin{aligned}
E\left\{\triangle_{2} E_{m}\right\}= & Q_{11}[E\{\cos \triangle \beta(t) \cos \triangle \alpha(t)\}-1]+Q_{21}[E\{\cos \triangle \beta(t) \operatorname{sen} \triangle \alpha(t)\}-1] \\
& +Q_{22}[E\{\cos \triangle \beta(t) \cos \triangle \alpha(t)\}-1]+Q_{12}[E\{\cos \triangle \beta(t) \operatorname{sen} \triangle \alpha(t)\}-1] \\
& +Q_{42}[E\{\operatorname{sen} \triangle \beta(t) \cos \triangle \alpha(t)\}-1]+Q_{31}[E\{\operatorname{sen} \triangle \beta(t) \cos \triangle \alpha(t)\}-1] \\
& +Q_{41}[E\{\operatorname{sen} \triangle \beta(t) \operatorname{sen} \triangle \alpha(t)\}-1]+Q_{51}+Q_{61}+Q_{71}+Q_{32}[E\{\operatorname{sen} \triangle \beta(t) \operatorname{sen} \triangle \alpha(t)\}-1] \\
& +Q_{82}+Q_{72}+Q_{52}+Q_{93}[E\{\cos \triangle \beta(t)\}-1]+Q_{10}[E\{\operatorname{sen} \triangle \beta(t)\}-1] .
\end{aligned}
$$

Os termos $Q_{i j}$ são quadraturas em seno e cosseno. Além disso, consideramos que os efeitos das velocidades no intervalo entre os desvios mínimos e máximos ao mesmo tempo são, praticamente, balanceados, porque os desvios ocorrem entre estes valores. Assim, os valores das velocidades com e sem os desvios, possuem, na média mesmos valores. Ou seja,

$$
E\left\{V_{R, T, N}(t)\right\}=V_{R, T, N}\left(t_{1}\right)
$$

\section{A. Desvios não Correlacionados entre si}

Resta-nos, agora, analisar se os desvios em "pitch" e em "yaw" são, separadamente, correlacionados ou não. Ou seja, se um desvios em "pitch" incorporado na órbita, devido a aplicação do empuxo, num determinado instante, interfere no desvio em "pitch" num instante posterior, quando acionados novamente os propulsores. Além disso, não estamos admitindo qualquer correlação entre os desvios em "pitch" e em "yaw", por motivo de na prática estes desvios não interferirem um no outro. Neste trabalho adotaremos a distribuição de probabilidade uniforme para os desvios. A correlação entre os desvios em mesma direção foi estudada numericamente em [11] e foi tomada como sugestão de modelo para o desgaste do sistema propulsor. Para este caso, sem correlação, o cálculo da esperança é dado, por exemplo, como segue,

$$
\begin{aligned}
E\{\cos \triangle \alpha\} & =\frac{1}{2 \triangle \alpha_{\max }} \int_{-\triangle \alpha_{\max }}^{\triangle \alpha_{\max }} \cos [\triangle \alpha] d(\triangle \alpha)-1 \\
& =\left.\frac{1}{2 \triangle \alpha_{\max }} \operatorname{sen}[\triangle \alpha]\right|_{-\triangle \alpha_{\max }} ^{\triangle \alpha_{\max }}-1 \\
& =\frac{\operatorname{sen} \triangle \alpha_{\max }}{\triangle \alpha_{\max }}-1
\end{aligned}
$$

$E \operatorname{sen} \triangle \beta=E \operatorname{sen} \triangle \alpha=0$,
Neste caso, usamos novamente a esperança do produto como sendo igual ao produto das esperanças para grandezas não correlacionadas.

Finalmente, a esperança calculada para todos os termos é igual a,

$$
\begin{aligned}
& E\left\{\triangle_{2} E_{m}\right\}=C_{1} {\left[\frac{\operatorname{sen} \triangle \alpha_{\max }}{\triangle \alpha_{\max }} \frac{\operatorname{sen} \triangle \beta_{\max }}{\triangle \beta_{\max }}-1\right] } \\
& C_{2}\left[\frac{\operatorname{sen} \triangle \beta_{\max }}{\triangle \beta_{\max }}-1\right]+Q_{T 1}-Q_{10}-Q_{T 2}, \\
& E\left\{\triangle_{2} E_{m}\right\}=C_{1}\left[\sum_{m=0}^{\infty}(-1)^{m} \frac{\triangle \alpha_{\max }^{2 m}}{(2 m+1) !}\right] \times \\
& \times\left[\sum_{n=0}^{\infty}(-1)^{n} \frac{\triangle \beta_{\max }^{2 n}}{(2 n+1) !}\right] \\
&+C_{2}\left[\sum_{n=0}^{\infty}(-1)^{n} \frac{\triangle \beta_{\max }^{2 n}}{(2 n+1) !}\right]+Q_{T},
\end{aligned}
$$

As constantes $Q_{T}, C_{1}$ e $C_{2}$ são somas de quadraturas.

O cálculo da esperança para o lado esquerdo da equação nos fornece,

$$
\begin{aligned}
E\left\{\triangle_{2} E_{m}\left(t_{1}, t_{2}\right)\right\} & =E\left\{\frac{\mu m}{2 a\left(t_{2}\right)} \frac{-\mu m}{2 a^{\prime}\left(t_{2}\right)}\right\} \\
& =\frac{\mu m}{2 a^{2}\left(t_{2}\right)} E\left\{\frac{\triangle a\left(t_{2}\right)}{a^{\prime 2}\left(t_{1}\right)}\right\},
\end{aligned}
$$

Tomando a variação do semi-eixo maior como sendo $a\left(t_{2}\right)=$ $a^{\prime}\left(t_{2}\right)-a(t)$ e expandindo em torno da razão $a\left(t_{2}\right) / a(t)$, obtemos,

$$
\begin{aligned}
E\left\{\triangle_{2} E_{m}\left(t_{1}, t_{2}\right)\right\}= & \frac{\mu m}{2}\left[\frac{1}{a^{2}\left(t_{2}\right)} E\left\{\triangle a\left(t_{2}\right)\right\}-\frac{1}{a^{3}\left(t_{2}\right)} E\left\{\triangle^{2} a\left(t_{2}\right)\right\}\right. \\
& \left.+\frac{1}{2 ! a^{4}\left(t_{2}\right)} E\left\{\triangle^{3} a\left(t_{2}\right)\right\}-\frac{1}{3 ! a^{5}\left(t_{2}\right)} E\left\{\triangle^{4} a\left(t_{2}\right)\right\}+\ldots\right] \\
& =\frac{\mu m}{2} \sum_{n=1}^{\infty}(-1)^{n+1} \frac{1}{(n-1) ! a^{n+1}\left(t_{2}\right)} E\left\{\triangle^{n} a\left(t_{2}\right)\right\}
\end{aligned}
$$


por,

$$
\sum_{n=1}^{\infty}(-1)^{n+1} \frac{1}{(n-1) ! a^{n+1}\left(t_{2}\right)} E\left\{\triangle^{n} a\left(t_{2}\right)\right\}=\left\{C_{4}+C_{3} \sum_{n=0}^{\infty}(-1)^{n} \frac{\triangle \alpha_{\max }^{2 n}}{(2 n+1) !}\right\} \sum_{n=0}^{\infty}(-1)^{n} \frac{\triangle \beta_{\max }^{2 n}}{(2 n+1) !}+C_{5}
$$

onde,

$$
C_{3}=\frac{2 C_{1}}{\mu m} ; \quad C_{4}=\frac{2 C_{2}}{\mu m} ; \quad C_{5}=\frac{2 Q_{T}}{\mu m}
$$

Expandindo o lado direito da equação (31) até termos de $6^{\mathrm{a}}$ ordem nos desvios, temos:

$$
\begin{aligned}
& E\left\{\triangle_{2} E_{m}\left(t_{1}, t_{2}\right)\right\}=C_{7}-\frac{C_{3}}{3 !}\left(\triangle \alpha_{\max }^{2}+\triangle \beta_{\max }^{2}\right)+\frac{1}{5 !}\left(C_{3} \triangle \alpha_{\max }^{4}+C_{6} \triangle \beta_{\max }^{4}\right) \\
& -\frac{1}{7 !}\left(C_{3} \triangle \alpha_{\max }^{6}+C_{6} \triangle \beta_{\max }^{6}\right)+\frac{1}{(3 !)^{2}}\left(\triangle \alpha_{\max } \triangle \beta_{\max }\right)^{2}+\frac{1}{(5 !)^{2}}\left(\triangle \alpha_{\max }^{2} \triangle \beta_{\max }^{2}\right)^{2} \\
& +\frac{1}{(7 !)^{2}}\left(\triangle \alpha_{\max }^{3} \triangle \beta_{\max }^{3}\right)^{2}-\frac{C_{3}}{(3 ! 5 !)}\left(\triangle \alpha_{\max }^{2} \triangle \beta_{\max }^{4}+\triangle \alpha_{\max }^{4} \triangle \beta_{\max }^{2}\right) \\
& +\frac{C_{3}}{(3 ! 7 !)}\left(\triangle \alpha_{\max }^{2} \triangle \beta_{\max }^{6}+\triangle \alpha_{\max }^{6} \triangle \beta_{\max }^{2}\right)+\frac{C_{3}}{(5 ! 7 !)}\left(\triangle \alpha_{\max }^{4} \triangle \beta_{\max }^{6}+\triangle \alpha_{\max }^{6} \triangle \beta_{\max }^{4}\right)+\ldots
\end{aligned}
$$

Tomando desvios pequenos, coerentes com a faixa de interesse prático das missões espaciais (no máximo $2^{\circ}$ ), podemos desprezar os termos de ordem superior a dois, o que equivale a $n=0$. Neste caso, teremos a relação de causa e efeito de primeira ordem,

$$
E\left\{\triangle a\left(t_{2}\right)\right\} \approx K_{1}-K_{2} \triangle \alpha_{\max }^{2}-K_{2} \triangle \beta_{\max }^{2}
$$

A equação (34) é um parabolóide de revolução. Jesus e Santos em $[10,11]$ mostraram os resultados das simulações numéricas para o caso de desvios superpostos e encontraram um parabolóide de revolução deformado para quaisquer valores dos desvios em "pitch" e em "yaw" e avaliaram que para a faixa de interesse prático a deformação não ocorreria, resgatando apenas um parabolóide de revolução. Os gráficos das Figuras 2, 3, 4 e 5 reproduzidos no anexo deste artigo, mostram estes resultados para órbitas teórica $(T)$ e prática $(P)$, desvios sistemáticos $(S)$ e operacionais $(O)$ simuladas no referido trabalho.

Estes gráficos mostram a relação de causa e efeito numérica entre os desvios de direção do vetor empuxo aplicado durante a queima de combustível nas trajetórias de veículos espaciais e os semi-eixos maiores finais destas trajetórias. Claramente, pode ser observado que quando os desvios em "pitch" e em "yaw" são pequenos, a superfície é um parabolóide de revolução, enquanto que, quando estes desvios crescem e, neste caso fora da faixa de interesse prático das missões espaciais, o parabolóide de revolução é deformado. Estes resultados das simulações com o método de Monte Carlo são confirmados pela relação de causa e efeito algébrica encontrada em (33) caso geral e (34) caso de interesse prático. O caso geral mostra que a relação de causa e efeito é construída a partir de potências de ordem par nos desvios de direção, mostrando que os desvios em direção do vetor empuxo interferirão fortemente na órbita final do veículo espacial, quando eles assumem valores altos. Isto significaria uma perda do veículo e comprometeria os objetivos e sucesso da missão espacial.

\section{CONCLUSÕES}

Concluindo, podemos dizer que para manobras de transferência orbital sujeita a desvios no vetor empuxo em direções "pitch" e "yaw", superpostos e não correlacionados entre si, existe uma relação de causa e efeito com o valor médio do semi-eixo maior final. Esta relação é não linear, composta por termos de ordem par em "pitch" e "yaw", além dos termos cruzados entre eles. Esta relação é representada por uma superfície no espaço composto pelos desvios e o valor médio do semi-eixo maior. A superfície é um parabolóide de revolução, que é deformado por causa da perda de simetria entre os termos da expansão dos desvios. No caso de desvios na faixa de interesse prático das missões espaciais, a relação é um parabolóide de revolução regular. Os nossos resultados confirmam as previsões das simulações numéricas exatas e são aplicáveis a qualquer veículo espacial com dinâmica atuada por sistemas de propulsão. Além disso, a superfície encontrada é também uma superfície ótima que descreve a dinâmica de um veículo espacial sob influência das forças de gravidade e de propulsores não ideais, com o mínimo de combustível possível. 


\section{APÊNDICE - FIGURAS}

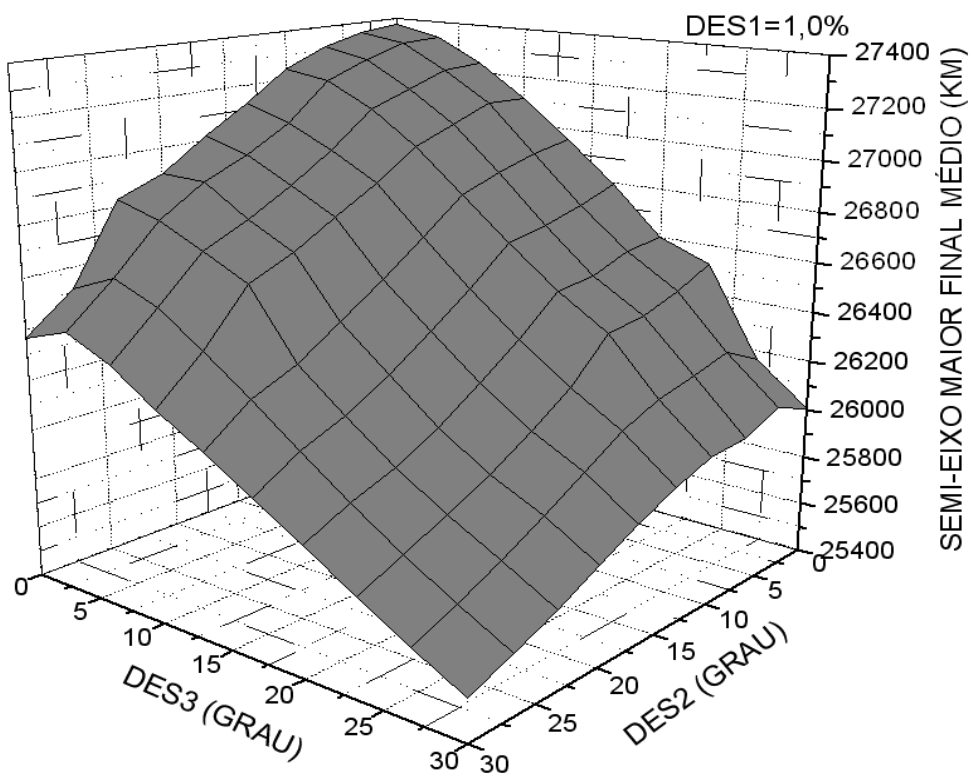

Fig. 2 - Relação de causa/efeito - Órbita PS

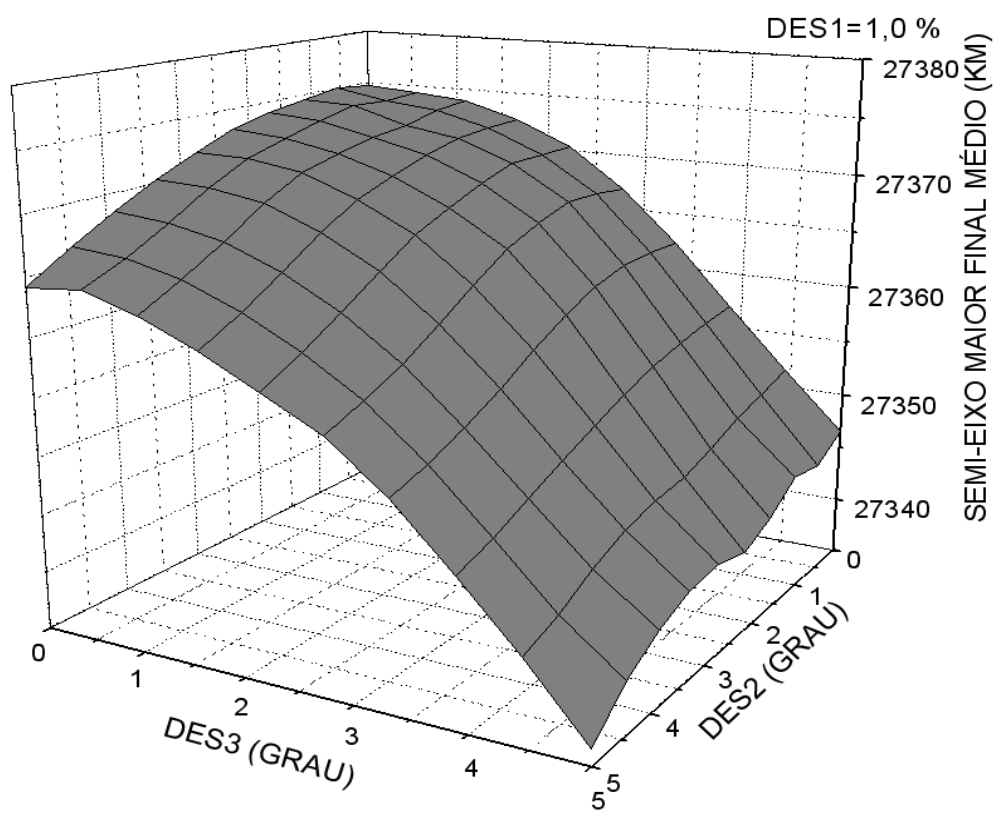

Fig. 3 - Relação de causa/efeito - Órbita PO 


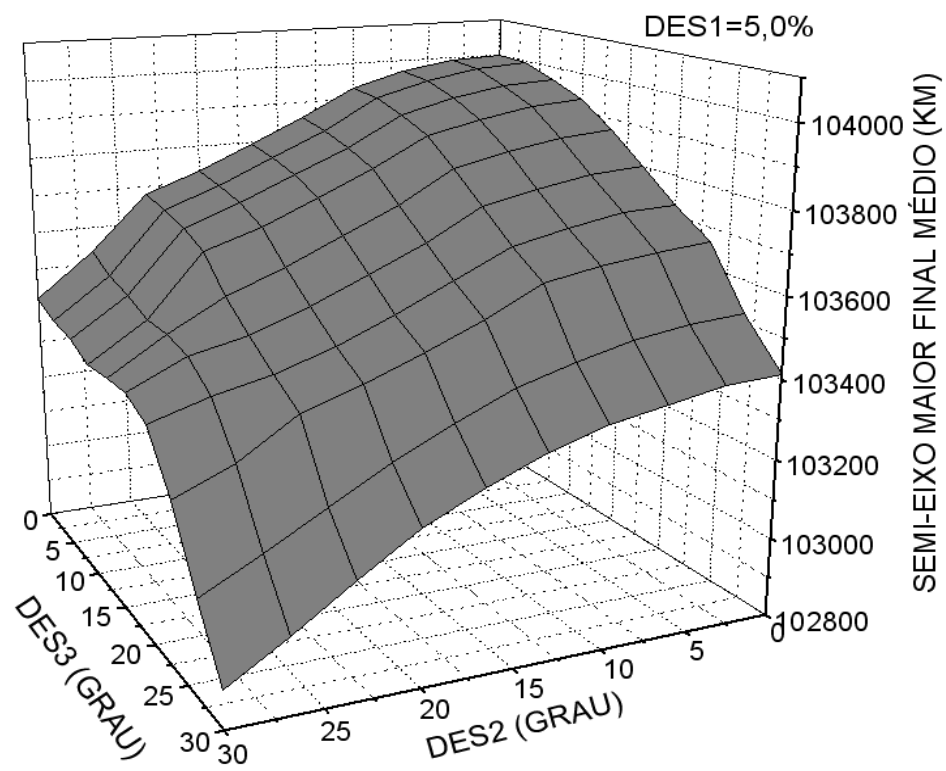

Fig. 4 - Relação de causa/efeito - Órbita TO

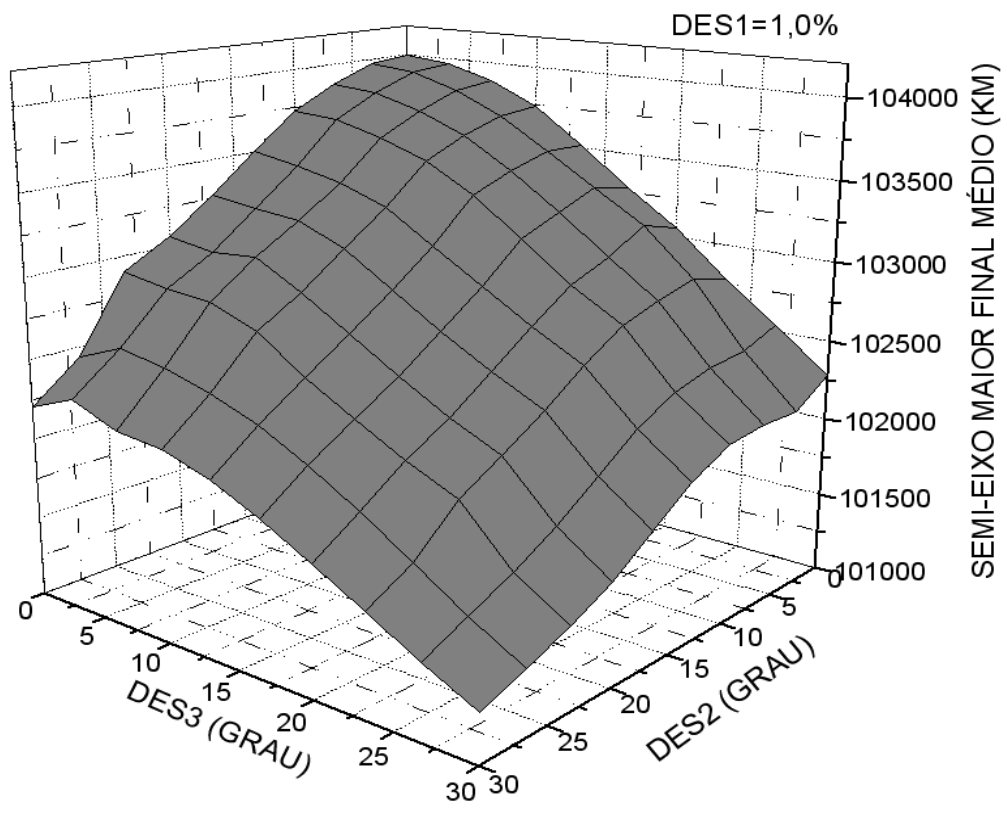

Fig. 5 - Relação de causa/efeito - Órbita TS

[1] R. H. Goddard; A method of reaching extreme altitudes, Smithsonian Inst. Publish. Misc. Collect 17 (2) (1919).

[2] W. Hohmann; Die erreichbarkeit der himmelkörper, Munique: s.e. $1 \mathrm{v},(1925)$.

[3] R. F. Hoelker and R. Silber; The Bi-Elliptic Transfer between circular co-planar orbits, Ballistic Missle Agency, Redstone Arsenal, Alabama, Army, EUA (1959).

[4] D. F. Lawden; Optimal programming of rocket thrust direction, Astronautica Acta 141 (1955).

[5] A.F.B.A. Prado; Análise, Seleção e Implementação de Procedimentos que Visem Manobras Ótimas de Satélites Artificiais, Dissertação de Mestrado. Não-publicada. INPE, São José dos Campos, São Paulo, Brasil (1989).

[6] D.L.F. Rodrigues; Análise Dinâmica da Transferência Orbital, Dissertação de Mestrado. Não-publicada. INPE, São
José dos Campos, São Paulo, Brasil (1991).

[7] M.M.N. Santos-Paulo; Estudo de Manobras Tridimensionais Impulsivas pelo Método de Altman e Pistiner, com Erros nos Propulsores, Dissertação de Mestrado. Não-publicada. INPE, São José dos Campos, São Paulo, Brasil (1998).

[8] W. Schultz; Transferências Biimpulsivas entre Órbitas Elípticas não Coplanares com Consumo Mínimo de Combustivel, Dissertação de Mestrado. Não-publicada. INPE, São José dos Campos, São Paulo, Brasil (1997).

[9] A.D.C. Jesus; Análise Estatística de Manobras Orbitais com Propulsão Finita Sujeita a Erros no Vetor Empuxo, Tese de Doutoramento. Não-publicada. INPE, São José dos Campos, São Paulo, Brasil (1999).

[10] A.D.C. Jesus, e F.M Santos; Continuous Trajectories under Superposition of the Thrust Vector Deviations, Adv. 
Space Dyn. - Celestial Mechanics and Astronautics 4 (1) 191 (2004).

[11] A.D.C. Jesus e F.M. Santos; Study about the Correlated Thrust Vector Deviations in Continuous Orbital Maneuvers, Adv. Space Dyn. - Celestial Mechanics and Astronautics, 4 (1) 180 (2004).
[12] A.D.C. Jesus, F.M. Santos, M.L.O. Souza e A.F.B.A. Prado; Study of the Nonimpulsive Orbital Maneuvers Feasibility due to the Restrictions of the Fuel Consumption and the Thrusters Power, Adv. Space Dyn. - Celestial Mechanics and Astronautics, 4 (1) 202 (2004). 